\title{
A consistent four-body CDCC model of low-energy reactions: Application to ${ }^{9} \mathrm{Be}+{ }^{208} \mathbf{P b}$
}

\author{
M. S. Hussein ${ }^{1,2,3}$, P. Descouvemont ${ }^{4}$ and L. F. Canto C $^{5,6}$
}

${ }^{1}$ Departamento de Física Matemática, Instituto de Física, Universidade de São Paulo, C.P. 66318, 05314-970, São Paulo, SP, Brazil

${ }^{2}$ Instituto de Estudos Avançados, Universidade de São Paulo, C.P. 72012, 05508-970, São Paulo, SP, Brazil

${ }^{3}$ Departamento de Física, Instituto Tecnológico de Aeronáutica, CTA, São José dos Campos, São Paulo, SP, Brazil

${ }^{4}$ Physique Nucléaire Théorique et Physique Mathématique, C.P. 229,

Université Libre de Bruxelles (ULB), B 1050 Brussels, Belgium

${ }^{5}$ Instituto de Física, Universidade Federal do Rio de Janeiro, C.P. 68528, 21941-972 Rio de Janeiro, RJ, Brazil

${ }^{6}$ Instituto de Física, Universidade Federal Fluminense, Av. Gal. Milton Tavares de Souza s/n, Niterói, RJ, Brazil

\begin{abstract}
We investigate the ${ }^{9} \mathrm{Be}+{ }^{208} \mathrm{~Pb}$ elastic scattering, breakup and fusion at energies around the Coulomb barrier. The three processes are described simultaneously, with identical conditions of calculations. The ${ }^{9} \mathrm{Be}$ nucleus is defined in an $\alpha+\alpha+n$ three-body model, using the hyperspherical coordinate method. We first analyze spectroscopic properties of ${ }^{9} \mathrm{Be}$, and show that the model provides a fairly good description of the low-lying states. The scattering with ${ }^{208} \mathrm{~Pb}$ is then studied with the Continuum Discretized Coupled Channel (CDCC) method, where the $\alpha+\alpha+n$ continuum is approximated by a discrete number of pseudostates. Optical potentials for the $\alpha+{ }^{208} \mathrm{~Pb}$ and $n+{ }^{208} \mathrm{~Pb}$ systems are taken from the literature. We present elasticscattering and fusion cross sections at different energies.
\end{abstract}




\section{Introduction}

Many experiments have been performed with the ${ }^{9} \mathrm{Be}$ nucleus, used as a target or as a projectile [1]. Although ${ }^{9} \mathrm{Be}$ is stable, it presents a Borromean structure, as the well known halo nucleus ${ }^{6} \mathrm{He}$. None of the two-body subsystems $\alpha+n$ or $\alpha+\alpha$ is bound in ${ }^{9} \mathrm{Be}$, which has important consequences on the theoretical description of this nucleus. Precise wave functions must include the three-body nature of ${ }^{9} \mathrm{Be}$. The hyperspherical formalism [2] is an ideal tool to describe three-body Borromean systems, as it does not assume a specific two-body structure, and considers the three particles $\alpha+\alpha+n$ on an equal footing.

In the present work [3], we aim at investigating ${ }^{9}$ Be scattering and fusion on a heavy target. The reaction framework is the Continuum Discretized Coupled Channel (CDCC) method (see Ref. [4] for a recent review), which is well adapted to weakly bound projectiles since it allows to include breakup channels. Going from two-body projectiles (such as $\mathrm{d}=\mathrm{p}+\mathrm{n}$ or ${ }^{7} \mathrm{Li}=\alpha+\mathrm{t}$ ) to three-body projectiles, however, strongly increases the complexity of the calculations, even if both options eventually end up with a standard coupledchannel system.

Many data have been obtained for ${ }^{9} \mathrm{Be}+{ }^{208} \mathrm{~Pb}$ elastic scattering $[5,6]$ and fusion $[7,8]$. These experimental data provide a good opportunity to test ${ }^{9} \mathrm{Be}$ wave functions. No assumption should be made about the cluster structure, and $\alpha+{ }^{208} \mathrm{~Pb}$ as well as $n+{ }^{208} \mathrm{~Pb}$ optical potentials are available in the literature.

\section{Three-body model of ${ }^{9} \mathrm{Be}$}

The determination of the ${ }^{9} \mathrm{Be}$ wave functions is the first step for the ${ }^{9} \mathrm{Be}+{ }^{208} \mathrm{~Pb} \mathrm{CDCC}$ calculation. For a three-body system, the Hamiltonian is given by

$$
H_{0}=\sum_{i=1}^{3} \frac{\boldsymbol{p}_{i}^{2}}{2 m_{i}}+\sum_{i<j=1}^{3} V_{i j}\left(\boldsymbol{r}_{i}-\boldsymbol{r}_{j}\right),
$$

where $\boldsymbol{r}_{i}$ and $\boldsymbol{p}_{i}$ are the space and momentum coordinates of the three particles with masses $m_{i}$, and $V_{i j}$ a potential between nuclei $i$ and $j$. For the $\alpha+\alpha$ interaction, we use the deep potential of Buck et al. [9]. The $\alpha+\mathrm{n}$ interaction is taken from Kanada et al. [10]. Both (real) potentials accurately reproduce the $\alpha+\alpha$ and $\alpha+$ nucleon phase shifts over a wide energy range. 
With these potentials, the ${ }^{9} \mathrm{Be}$ ground state is too bound $(-3.12 \mathrm{MeV}$, while the experimental value is $-1.57 \mathrm{MeV}$ with respect to the $\alpha+\alpha+n$ threshold). We have therefore introduced a phenomenological three-body force which reproduces the experimental binding energy of the $3 / 2^{-}$ground state. The r.m.s. radius, the quadrupole moment, and the magnetic moment are $\sqrt{r^{2}}=2.36 \mathrm{fm}, Q\left(3 / 2^{-}\right)=4.96 e . \mathrm{fm}^{2}$, and $\mu=-1.33 \mu_{N}$, respectively. These values are in fair agreement with experiment $(2.45 \pm 0.01 \mathrm{fm}, 5.29 \pm$ 0.04 e. $\mathrm{fm}^{2}$ and $-1.18 \mu_{N}$, respectively)

\section{The CDCC method}

We present here a brief outline of the CDCC method, and we refer to Ref. [11] for specificities of three-body projectiles. The CDCC method is based on approximate solutions of the projectile Hamiltonian (1)

$$
H_{0} \Phi_{k}^{j m \pi}=E_{0, k}^{j \pi} \Phi_{k}^{j m \pi}
$$

where $k$ are the excitation levels in partial wave $j \pi$. Solutions with $E_{0, k}^{j \pi}<0$ correspond to bound states of the projectile, whereas $E_{0, k}^{j \pi}>0$ correspond to narrow resonances or to approximations of the three-body continuum. These states cannot be associated with physical states, and are referred to as pseudostates. The ${ }^{9} \mathrm{Be}$ spectrum used in the present model is shown in Fig. 1.

The Hamiltonian of the projectile + target system is written as

$$
H(\boldsymbol{R}, \boldsymbol{x}, \boldsymbol{y})=H_{0}(\boldsymbol{x}, \boldsymbol{y})-\frac{\hbar^{2}}{2 \mu_{P T}} \Delta_{R}+V(\boldsymbol{R}, \boldsymbol{x}, \boldsymbol{y})
$$

where $\mu_{P T}$ is the reduced mass of the system, and $R$ the relative coordinate. The Jacobi coordinates $\boldsymbol{x}$ and $\boldsymbol{y}$ are proportional to $\boldsymbol{r}_{\mathrm{Be}-\mathrm{n}}$ and $\boldsymbol{r}_{\alpha-\alpha}$, respectively. The potential term reads

$$
V(\boldsymbol{R}, \boldsymbol{x}, \boldsymbol{y})=V_{1 t}(\boldsymbol{R}, \boldsymbol{y})+V_{2 t}(\boldsymbol{R}, \boldsymbol{x}, \boldsymbol{y})+V_{3 t}(\boldsymbol{R}, \boldsymbol{x}, \boldsymbol{y})
$$

where the three components $V_{i t}$ are optical potentials between fragment $i$ and the target.

In order to solve the Schrödinger equation associated with (3), the total wave function with angular momentum $J$ and parity $\Pi$ is expanded as

$$
\Psi_{(\omega)}^{J M \Pi}(\boldsymbol{R}, \boldsymbol{x}, \boldsymbol{y})=\sum_{j \pi k L} \varphi_{j \pi k L}^{J M \Pi}\left(\Omega_{R}, \boldsymbol{x}, \boldsymbol{y}\right) g_{j k L(\omega)}^{J \Pi}(R),
$$




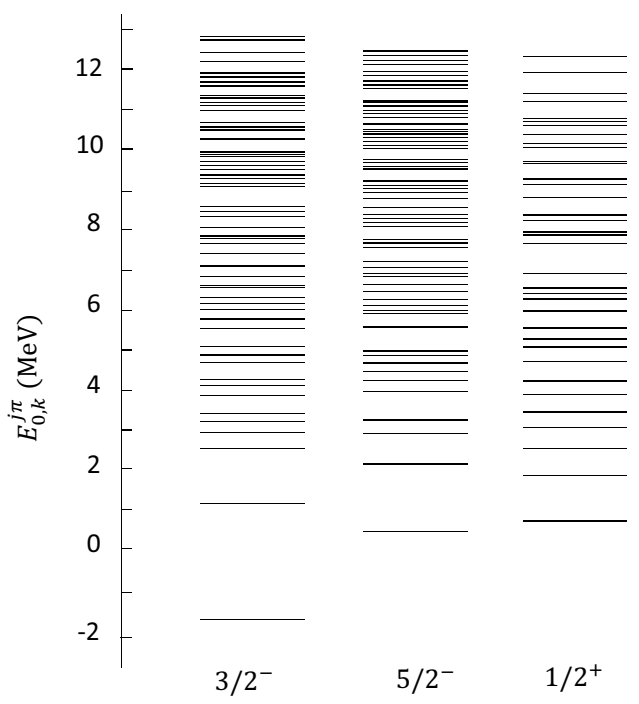

Figure 1: Pseudostate energies (defined from the $\alpha+\alpha+n$ threshold) for the dominant partial waves $j=3 / 2^{-}, 5 / 2^{-}, 1 / 2^{+}$.

where $\omega$ is the entrance channel, and where the channel wave functions are given by

$$
\varphi_{j \pi k L}^{J M \Pi}\left(\Omega_{R}, \boldsymbol{x}, \boldsymbol{y}\right)=i^{L}\left[\Phi_{k}^{j \pi}(\boldsymbol{x}, \boldsymbol{y}) \otimes Y_{L}\left(\Omega_{R}\right)\right]^{J M} .
$$

The radial functions $g_{c}^{J \Pi}(R)$ (we use the notation $c=(j, \pi, k, L)$ ) are obtained from the system

$$
\left[-\frac{\hbar^{2}}{2 \mu_{P T}} \frac{d^{2}}{d R^{2}}+E_{c}-E\right] g_{c(\omega)}^{J \Pi}(R)+\sum_{c^{\prime}} V_{c, c^{\prime}}^{J \Pi}(R) g_{c^{\prime}(\omega)}^{J \Pi}(R)=0
$$

where $E$ is the c.m. energy, and where the coupling potentials are given by matrix elements

$$
V_{c, c^{\prime}}^{J \Pi}(R)=\left\langle\varphi_{c}^{J M \pi}|V| \varphi_{c^{\prime}}^{J M \pi}\right\rangle+\frac{\hbar^{2}}{2 \mu_{P T}} \frac{L(L+1)}{R^{2}} \delta_{c c^{\prime}} .
$$

As the fragment-target potentials (4) are optical potentials, matrix elements (8) contain a real and an imaginary parts (see Ref. [3] for detail). We solve Eq. (7) with the $R$-matrix theory [12]. 


\section{Application to the ${ }^{9} \mathrm{Be}+{ }^{208} \mathrm{~Pb}$ system}

\subsection{Elastic scattering}

The calculations are performed with the $j^{\pi}=3 / 2^{ \pm}, 5 / 2^{ \pm}, 1 / 2^{ \pm}$partial waves on ${ }^{9} \mathrm{Be}$, and the cutoff energy is $12.5 \mathrm{MeV}$. Figure 2 shows ${ }^{9} \mathrm{Be}+{ }^{208} \mathrm{~Pb}$ elastic cross sections at two typical energies (the data are taken from Refs. $[5,13]$ ). In contrast with optical-model calculations [5], no renormalization of the potential is needed. The absorption is simulated by the imaginary parts of the $\alpha-{ }^{208} \mathrm{~Pb}$ and $\mathrm{n}-{ }^{208} \mathrm{~Pb}$ interactions, and by the $\alpha+\alpha+n$ discretized continuum. Calculations involving only the $3 / 2^{-}$ground state are shown as dashed lines, and the full calculations as solid lines. From Fig. 2, it appears that continuum couplings are more important at low energies. At $E=38 \mathrm{MeV}$, the single-channel calculation, limited to the ${ }^{9} \mathrm{Be}$ ground state is significantly different from the data. This confirms the conclusion of a previous work [14] which suggests that continuum couplings are more important at low energies.

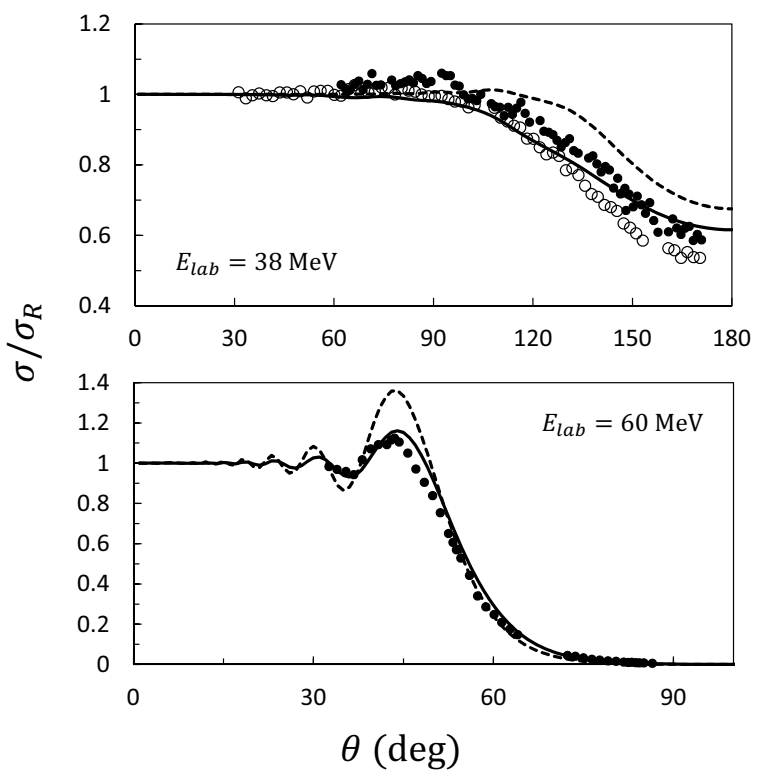

Figure 2: ${ }^{9} \mathrm{Be}+{ }^{208} \mathrm{~Pb}$ elastic cross sections (divided by the Rutherford cross section) at two ${ }^{9}$ Be laboratory energies, and for different sets of ${ }^{9} \mathrm{Be}$ partial waves. The experimental data are taken from Ref. [5] (filled circles) and Ref. [13] (open circles). 


\subsection{Fusion}

The ${ }^{9} \mathrm{Be}+{ }^{208} \mathrm{~Pb}$ fusion reaction has been studied theoretically by several groups (see Ref. [15] and references therein). Data about the various mechanisms (total TF, complete $\mathrm{CF}$ and incomplete ICF fusion) are also available in the literature $[7,8,16]$.

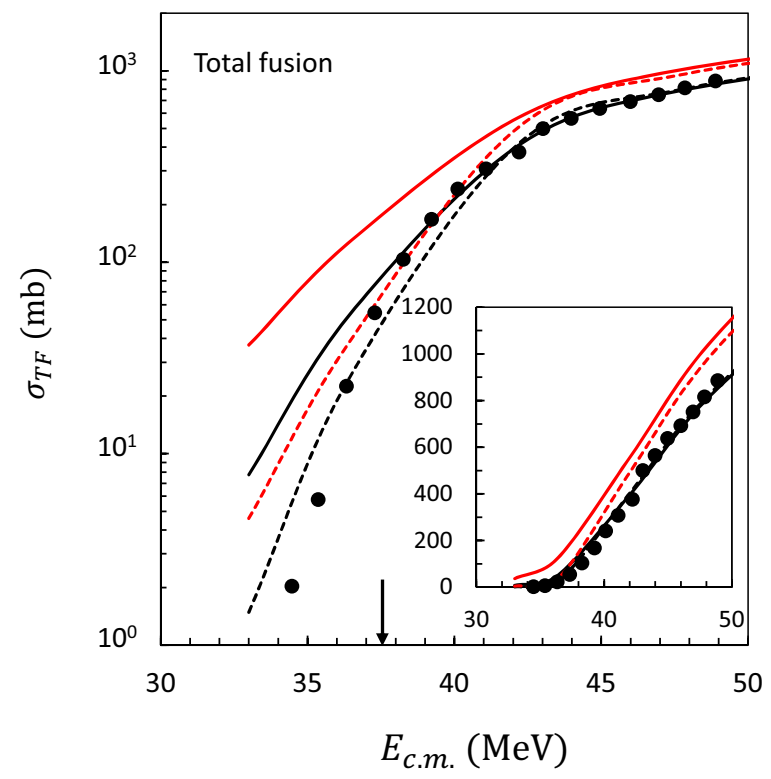

Figure 3: Total fusion cross sections with (black lines) and without (red lines) removal of the neutron capture. The dashed lines represent the single-channel calculations, and the solid lines represent the full calculations. The Coulomb barrier energy $V_{B}$ is indicated by a vertical arrow.

The total fusion cross section essentially involves two contributions: the complete fusion, where the whole projectile is absorbed, and the incomplete fusion, where only a part of the projectile charge is absorbed by the target. As far as the total fusion is concerned, the cross section can be obtained by using the scattering matrices. The CDCC total fusion cross sections obtained in this way are shown in Fig. 3 as red lines. Above the Coulomb barrier, the present calculation overestimates the data by about a factor two. This overestimation is due to the different definitions adopted for fusion in the experiment and in the calculation. In other words, the theory defines fusion as the absorption of the whole projectile or any of its fragments, including the neutron. On the other hand, the experimental cross section only includes contributions from the absorption of charge [7]. Thus, the two 
cross sections differ by the neutron absorption which, owing to the lack of a Coulomb barrier, may be quite large.

The CDCC calculation can be adapted to the experimental conditions by using an equivalent definition of the fusion cross section, and by removing the neutron-target imaginary potential. The results obtained in this way are shown in Fig. 3 as black lines. By excluding the neutron capture, we obtain an excellent agreement with experiment above $E_{\mathrm{cm}} \approx 40 \mathrm{MeV}$. This is more clearly shown in the inset, drawn in a linear scale. We confirm previous CDCC calculations $[15,21]$ which conclude that the influence of breakup channels increases at sub-barrier energies. Below the Coulomb barrier, the single-channel calculation is in better agreement with experiment, and the full calculation overestimates the data. This discrepancy is common to most CDCC fusion calculations (see, for example, Fig. 1 of Ref. [15]), and deserves further investigation.

\section{Conclusion}

We have applied the CDCC formalism to the ${ }^{9} \mathrm{Be}+{ }^{208} \mathrm{~Pb}$ system. In a first step, we have computed ${ }^{9} \mathrm{Be}$ wave functions in a three-body $\alpha+\alpha+n$ model. These calculations were performed for low-lying states, but also for pseudostates, which represent positive-energy approximations of the continuum. The main advantage of the hyperspherical approach is that it treats the three-body continuum without any approximation concerning possible ${ }^{8} \mathrm{Be}+n$ or ${ }^{5} \mathrm{He}+\alpha$ cluster structures, which are in fact strictly equivalent.

The three-body model of ${ }^{9} \mathrm{Be}$ relies on $\alpha+\alpha$ and $\alpha+n$ (real) interactions, which reproduce very well the elastic phase shifts. With these bare interactions, the ${ }^{9} \mathrm{Be}$ ground state is slightly too bound. We therefore introduce a phenomenological three-body force to reproduce the experimental ground-state energy. The spectroscopic properties of low-lying states is in fair agreement with experiment.

We used first the ${ }^{9} \mathrm{Be}$ wave functions in a CDCC calculation of the ${ }^{9} \mathrm{Be}+{ }^{208} \mathrm{~Pb}$ elastic scattering at energies close to the Coulomb barrier. As expected, including continuum channels improves the theoretical cross sections. A fair agreement with the data is obtained.

We also applied the four-body CDCC to the ${ }^{9} \mathrm{Be}+{ }^{208} \mathrm{~Pb}$ fusion, by using the same potentials as for elastic scattering. The total fusion cross section is found in good agreement with experiment, except at very low energies, where we overestimate the data. 


\section{References}

[1] N. Keeley et al., Prog. Part. Nucl. Phys. 63, 396 (2009)

[2] M.V. Zhukov et al., Phys. Rep. 231, 151 (1993)

[3] P. Descouvemont et al., Phys. Rev. C 91, 024606 (2015)

[4] M. Yahiro et al., Prog. Theor. Phys. Supp. 196, 87 (2012)

[5] R.J. Woolliscroft et al., Phys. Rev. C 69, 044612 (2004)

[6] S.K. Pandit et al., Phys. Rev. C 84, 031601 (2011)

[7] M. Dasgupta et al., Phys. Rev. Lett. 82, 1395 (1999)

[8] M. Dasgupta et al., Phys. Rev. C 70, 024606 (2004)

[9] B. Buck, H. Friedrich, C. Wheatley, Nucl. Phys. A 275, 246 (1977)

[10] H. Kanada et al., Prog. Theor. Phys. 61, 1327 (1979)

[11] M. Rodríguez-Gallardo et al., Phys. Rev. C 77, 064609 (2008)

[12] P. Descouvemont, D. Baye, Rep. Prog. Phys. 73, 036301 (2010)

[13] N. Yu et al., J. Phys. G 37, 075108 (2010)

[14] T. Druet, P. Descouvemont, Eur. Phys. J. A 48, 147 (2012)

[15] V. Jha, V.V. Parkar, S. Kailas, Phys. Rev. C 89, 034605 (2014)

[16] M. Dasgupta et al., Phys. Rev. C 81, 024608 (2010)

[17] L.F. Canto et al., Phys. Rep. 424, 1 (2006); Phys. Rep. 596, 1 (2015)

[18] K. Hagino, N. Takigawa, Prog. Theor. Phys. 128, 1001 (2012)

[19] L.F. Canto, M.S. Hussein, Scattering Theory of Molecules, Atoms and Nuclei (World Scientific Publishing, Singapore, 2013)

[20] A. Diaz-Torres, I.J. Thompson, Phys. Rev. C 65, 024606 (2002)

[21] A. Diaz-Torres, I.J. Thompson, C. Beck, Phys. Rev. C 68, 044607 (2003) 